Folia Histórica del Nordeste, $\mathbf{N}^{\mathbf{0}} 21$ (Resistencia, 2013) IIGHI, IH - CONICET, UNNE

\title{
NUEVOS APORTES DE LA LINGÜÍSTICA PARA LA INVESTIGACIÓN DE DOCUMENTOS EN GUARANÍ DE LA ÉPOCA COLONIAL (SIGLO XVIII)
}

\author{
New Linguistic Contributions to Research on Guarani Manuscripts of the \\ Colonial Age (18th Century)
}

\section{Leonardo Cerno y Franz Obermeier}

\section{Resumen}

En este trabajo presentamos y describimos un conjunto de manuscritos procedentes de las Reducciones Jesuíticas de la Provincia del Paraguay de la época colonial, recientemente hallados y prácticamente desconocidos (o dados por perdidos). Los manuscritos están redactados íntegramente en guaraní y, como parte de la producción escrita de las Misiones, presentan aspectos de interés tanto para la lingüística como para la historia y la antropología del Río de la Plata colonial. Para establecer una función de estos documentos analizamos el complejo sociocultural reduccional, particularmente las prácticas y significaciones vinculadas con la escrituralidad del guaraní. Nuestra conclusión es que los manuscritos que presentamos pueden llegar a cambiar nuestra comprensión del complejo dialectal del guaraní así como contribuir con mejores datos a una "visión indígena" del proceso reduccional.

$<$ Misiones Jesuíticas $><$ manuscritos $><$ variación lingüística $><$ guaraní $>$

\begin{abstract}
The present article examines a certain number of manuscripts which are newly discovered or rediscovered after a long lapse of time, all written in the Jesuit reductions of the Paraguay Province in colonial times. The manuscripts are entirely in Guarani and as testimony of the literacy in the reductions they are of equal importance for linguists, historians and anthropologists working about the La Plata region. The question is to determine which function these documents had in the socio-cultural complex of the Jesuit missions, especially in relation with the social practices, the indigenous literacy and its significance. We conclude pointing out that these manuscripts can help us to change our understanding about the Guarani dialects and present us some major sources for an "indigenous vision" on the Jesuit reductions to be reconstructed in critical discussion of earlier research patterns.
\end{abstract}

$<$ Jesuit Reductions of Paraguay $><$ Manuscripts $><$ Linguistic Variation $><$ Guaraní $>$ 


\section{Introducción y antecedentes}

La investigación referida a las Misiones Jesuíticas del Paraguay (1609-1768) trabaja sobre el análisis de documentos y monumentos vinculados a ese período histórico y su contexto. En lo que respecta a las fuentes documentales, junto al vasto conjunto de textos producidos por los jesuitas en las Misiones, llaman también la atención los documentos escritos en lengua indígena. Los comienzos de la literatura ${ }^{1}$ en guaraní coinciden con los orígenes mismos de la Misión, empresa que exigió la traducción de catecismos, oraciones y sermones a la lengua de los nativos. La literatura religiosa fue escrita casi en su totalidad por los padres jesuitas y ocupa la mayor parte del corpus conocido en guaraní reduccional (Melià 1992: 112). A esta producción debemos sumar, en etapa posterior al siglo XVIII, los textos escritos por indígenas, dominio en el que existe una diversificación mayor de géneros (cartas, actas de cabildos, narrativa histórica, etc.) (Cf. Neumann 2007: 54 y ss). En los últimos años, los más interesados en el estudio y comprensión de estos documentos indígenas no han sido los lingüistas, sino los historiadores y antropólogos. Este hecho se explica en parte porque el trabajo del lingüista obliga al conocimiento de la lengua nativa, en este caso copiosa y difícil, sin hablantes nativos y hermética incluso para los hablantes del moderno guaraní paraguayo (Cf. Melià 1969 [2003]: 325, Neumann 2005: 47, Thun 2008a: 219). Los historiadores y antropólogos, por su parte, han llevado a cabo estudios de fuentes indígenas en guaraní en vistas a interpretar el proceso colonial y reduccional desde la "perspectiva de los indígenas" (Ganson 2003, Neumann 2005 y 2007, Wilde 2012). No obstante se presenta aquí un inconveniente obvio: tales estudios se limitan a los textos ya traducidos al español (o al portugués), quedando fuera del alcance un conjunto significativo de documentos todavía no traducidos, cuya cantidad y calidad ha sido reconocida por varios expertos en historia reduccional (Pastells 1918-49 Furlong 1953, Melià 1992: 135-156; 2006). Por otro lado, el trabajo con traducciones constriñe al investigador a la función informativa del mensaje, quedando fuera su alcance los significados latentes vinculados con la semántica y pragmática del discurso indígena. ¿Cómo podría una "filología guaraní" contribuir a esta problemática? Es cierto que esta filología ya existe y que ha sido fundada, en su vertiente moderna, por Bartomeu Melià, cuya obra constituye la puerta de entrada a una visión histórico-lingüística de la sociedad reduccional (Melià 1969 [2003], 1992, 2011). Pero esta obra presenta, a nuestro modo de ver, el inconveniente de estar centrada en la "perspectiva jesuítica" del guaraní, es decir en la lengua normatizada por estos y documentada en los diferentes textos religiosos y metalingüísticos, dejando de lado aspectos relativos al discurso indígena, de vital importancia para la antropología histórica. A nuestro modo de ver, una filología guaraní capaz de contribuir a la historia y la antropología debe partir de una concepción de lenguaje que contemple la diversidad lingüística interna de

\footnotetext{
${ }^{1}$ Usamos el término "literatura" en sentido general, para referirnos a la práctica de escritura y lectura de textos. El concepto de literatura tal como lo conocemos actualmente, implicando las nociones de „originalidad“ y ,autoría“, no surgió sino hacia mediados del siglo XVIII y no sería pertinente referirse con él en sentido estricto a la producción de las Misiones Jesuíticas.
} 
esta comunidad y la organización de los medios expresivos (variedades y estilos del habla) en la actividad lingüística concreta. Nuestro artículo tiene por objetivo sentar las bases para la investigación de la literatura en guaraní atenta a estos principios. Nuestro objetivo se persigue a partir de un ejemplo concreto: el análisis de nuevos documentos que acrecentan el acervo de la literatura en lengua indígena en las Reducciones. Se trata de un conjunto de nuevos y valiosos manuscritos en guaraní recientemente descubiertos por los investigadores de la Universidad de Kiel (Alemania) y elaborados en el marco del Proyecto PeKY (Proyecto Kuatia Ymaguare) del cual algunos avances ya han sido presentados (Cerno 2010, Obermeier 2011, Thun en prensa). Los textos a que nos referimos son, por un lado, dos manuscritos fundamentales, el llamado Manuscrito Gülich, y el texto conocido como Diario hecho por un indio de lo que sucedió en el segundo desalojamiento de los portugueses [...] o como Diario del desalojo de 1704-05. Por el otro lado, nos referimos a algunos textos de medicina vernácula. Nuestra hipótesis en sentido general es que las prácticas de escrituralidad en guaraní se organizaron en torno a la valoración del habla coloquial de los indígenas por parte de los jesuitas, estrato social más próximo al control de los medios de la escritura. Nuestra hipótesis en sentido particular es que durante la primera mitad del siglo XVIII, época en que ubicamos la elaboración de los documentos detallados arriba, asistimos a un cambio en la actitud de los jesuitas hacia la diversidad lingüística de la comunidad, hecho que conduce a una nueva la valoración del discurso indígena.

En la primera parte presentamos y describimos los manuscritos recientemente hallados y algunas de las preguntas que a partir de aquí se plantean. En la segunda parte ofrecemos una reinterpretación del proceso de escrituralidad en guaraní en las Reducciones tal que permita explicar de manera coherente el significado y función de los mencionados documentos, así como proporcionar una visión sistemática de conjunto de la literatura en guaraní jesuítico.

\section{Nuevos documentos en el horizonte de la literatura reduccional}

Para entablar una discusión sobre algunos aspectos relevantes de estos manuscritos remitimos a la tipología de Harald Thun sobre las prácticas de escrituralidad en guaraní (Thun 2003). Este autor distingue entre una escrituralidad propia del ámbito de "lo eterno", cuyo ejemplo es la literatura cristiana elaborada por los jesuitas, y cuyo uso corresponde a la práctica religiosa, y una escrituralidad del ámbito "de lo temporal", de uso en la vida práctica, económica, política y administrativa de las Reducciones, y cuyo ejemplo comprende los documentos escritos por mayordomos, cabildantes y caciques indígenas, particularmente con posterioridad al siglo XVIII. (Cf. también Thun 2008a, 2008b). Con respecto al trabajo indígena en el proceso de la escritura, el autor distingue dos etapas: la escrituralidad "guiada y controlada", en que el indio escribe bajo la iniciativa y el control de un regular de la Compañía de Jesús, y la etapa de "liberación de la escrituralidad", etapa que irrumpe con la eclosión de las cartas indígenas de la época del Tratado de Madrid (1750) y las guerras guaraníticas 
(1754-1756). A continuación presentamos tres documentos recientemente hallados que acrecentan el corpus existente. En conjunto, se trata de documentos que habían sido mencionados en bibliografías del siglo XIX (p.ej. De Ángelis 1853, Apéndice), pero que hasta hoy se daban por perdidos

\subsection{El manuscrito Gülich}

Una colección de textos diversos, no todos completos, escritos íntegramente en lengua guaraní, en forma de diálogos sobre la vida cotidiana en las Misiones, constituyen el llamado Manuscrito Gülich. El volumen suma en conjunto unas 150 páginas. Es difícil establecer claramente la época y lugar de composición de los textos, así como sus posibles autores y su uso o finalidad dentro de las Reducciones. El texto, cuyas últimas menciones constaban en bibliografías del siglo XIX, se había dado por perdido hace más de cien años. La copia que poseemos es del siglo XIX. Se trata de una copia hecha a pedido por Julio Platzmann, conocido investigador alemán de lenguas indígenas, a instigación del emperador Pedro II del Brasil, y cuyo mediador en esta tarea es Carl Henning. El original del manuscrito perteneció a Friedrich von Gülich (1820, Osnabrück - 1903, Wiesbaden). Gülich fue un diplomático prusiano en los estados del Plata hacia 1857, y su gestión más importante fue un tratado de libre comercio con la Confederación de estados argentinos. Durante su viaje a la región, Gülich habría obtenido el manuscrito que hoy en día identificamos con su nombre. Es difícil saber los detalles de la adquisición, pero es muy probable que haya ocurrido a través de la compra del mismo de la colección de De Ángelis. El documento es presumiblemente mencionado como "Diálogos en lengua guaraní" en el Apéndice a la Colección de obras impresas y manuscritas que tratan principalmente del Río de la Plata, impreso en pocos ejemplares cerca de 1854. Ese Apéndice menciona los manuscritos e impresos en lenguas indígenas que pertenecieron a De Ángelis, a quien Gülich conocía. El original del Manuscrito Gülich no ha sido encontrado, probablemente fue destruido con la colección de la familia Gülich durante la Segunda Guerra Mundial. Sí hay copias hechas en el siglo XIX, de las cuales una es la que ha llegado a nuestras manos. Por las bibliografías de la época sabemos que hubo probablemente dos copias, una hecha para el Emperador del Brasil, y otra que, a través del lingüista brasileño Baptista Caetano de Almeida Noguera, acabó en la colección del lingüista norteamericano Daniel Brinton. Es la copia perteneciente a esta última colección la que fue descubierta recientemente por Franz Obermeier, coautor de este artículo (ver para más detalles Obermeier 2011).

Ante los primeros exámenes del texto por parte de los investigadores del proyecto $P e K Y$, el manuscrito reveló su importancia por el hecho de ser, dentro del corpus del guaraní jesuítico conocido, uno de los más representativos de la vertiente "de lo temporal". Constan aquí ricas referencias a asuntos llanos como la preparación de comidas, el trabajo en las estancias, las pautas de buena conducta en las Reducciones, la educación de los niños, y otros. Su estructura dialógica y su registro coloquial permitirán plantear hipótesis acerca del uso y función de estos textos en el marco reduccional, pero 
quedan todavía por resolver cuestiones relativas al proceso de producción del texto: p.ej. si fue escrito por indígenas bajo la supervisión de los jesuitas, o por jesuitas con la ayuda de indígenas. Acerca de su función, dada su estructura dialógica, se plantean preguntas acerca de si estos diálogos fueron escritos para ser representados, en forma de "sainetes" o teatro no religioso, o si fueron concebidos para el aprendizaje de la lengua por parte de los nuevos misioneros llegados de Europa, mediante el método de la repetición y el diálogo. Más adelante discutiremos algunos de estos supuestos. La traducción y un estudio crítico del documento se encuentra en estado avanzado.

\subsection{Diario del desalojo de 1704-1705}

Otro texto prácticamente desconocido es el llamado Diario del desalojo de 1704-1705. Con excepción de una referencia en el mencionado Apéndice de De Ángelis, que poseía el original, o en otras menciones que dependen del Apéndice, durante el siglo XIX no existen referencias al documento, con lo que éste desapareció prácticamente del horizonte historiográfico durante más de cien años. Quien lo redescubrió, o mejor dicho, dio a conocer su existencia es Bartomeu Melià, que obtuvo una copia de un ejemplar a su vez copiado y perteneciente a una colección privada (de Enrique A. Peña, hijo de Ernique Peña), donde el texto se encontraba antes de ser vendido. La ubicación actual del original es desconocida, sólo disponemos de una copia Xerox. Melià publicó algunos extractos traducidos al español (Melià 2000), incluyendo algunas interpretaciones en torno al contexto de producción del manuscrito. Para Melià el autor del texto es un secretario indígena, posiblemente de Corpus, que hace un "reporte" o "crónica" in situ de los acontecimientos ocurridos durante el segundo desalojo de la Colonia del Sacramento (Cf. Melià 2000, Melià 2006: 4-5). En el sitio de Colonia de Sacramento, llevado a cabo por los españoles entre 1704 y 1705, participaron guerreros guaraníes de las Misiones.

El Diario del desalojo posee formato de diario, y habría sido escrito durante o inmediatamente después del sitio de la Colonia del Sacramento. Consta de unas 52 páginas manuscritas, y relata los acontecimientos del sitio desde una perspectiva indígena, aunque todavía es discutible quién es su autor, es decir, si indígena o no. El sitio de Colonia de Sacramento está también documentado por otras fuentes españolas de la época: una relación del sitio fue publicada en Lima de 1705, y una relación historial, basada probablemente en cartas historiales, también aparece en Lima en 1705. Pero hasta hoy no hay menciones de que alguien haya utilizado el texto en guaraní, en los siglos subsiguientes, para el estudio historiográfico.

Uno de los problemas que presenta una adecuada interpretación del texto es la discusión sobre si se trata de escrituralidad indígena, y, dentro de este contexto, si conviene interpertarlo como escrituralidad "controlada", es decir, bajo la prescripción y supervición de los jesuitas que formaban parte del ejército español, o como escrituralidad "libre". Sabemos de las investigaciones de Neumann que hubo una literalidad o escrituralidad entre ciertos indígenas de las reducciones (Neumann 
2005: 56 y ss.). Ahora bien, la escrituralidad de los indígenas habría hecho eclosión hacia 1750, en la fase de la guerra guaranítica, época de una escritura aparentemente espontánea por parte de los indígenas que buscaban expresar su pensamiento político frente a las autoridades coloniales. En este panorama, un documento como el Diario del desalojo de 1704-1705, de una extensión tan llamativa (52 páginas) a principios del siglo XVIII, constituye un hecho excepcional. Debe pensarse que las noticias que tenemos acerca de cartas y memorias escritas por indígenas, o incluso de otras obras como las traducciones manuscritas al guaraní de la Conquista Espiritual de Montoya, son posteriores a 1720, y que un diario de esta naturaleza es excepcional dentro de este conjunto y en esta época concreta. ¿Sería esto un argumento para discutir las hipótesis de que el Diario fue escrito por un indígena de manera libre, y plantear más bien una planificación del texto por parte de las autoridades jesuitas o seculares, y una ejecución por parte de un secretario indígena y/o un padre jesuita? En el contexto de la escrituralidad jesuítica se puede pensar en una producción dirigida por los jesuitas, y también las numerosas correcciones que presenta el texto original hacen pensar en eso. Otra de las cuestiones es resolver quién estaría interesado en hacer describir, con tantos pormenores, y en conservar, un diario tan detallado de ese sitio militar, y para qué uso ulterior. Como otra de las preguntas que el texto plantea, las respuestas que pueden darse son todavía preliminares. La traducción, edición cuidada del texto y su adecuada contextualización es lo que permitirá aproximarnos con más certidumbres a estos materiales.

\subsection{Textos de medicina}

A pesar de existir muchos estudios de historia de la medicina colonial, se sabe muy poco sobre la contribución de la medicina indígena a la medicina española del Río de la Plata. El célebre Materia médica misionera, del hermano Pedro de Montenegro, es uno de los referentes en este contexto de estudios. Se trata de un texto conocido desde hace tiempo, existiendo una edición ya en 1945, editada por Raúl Quintana, y basada en uno de los manuscritos que habían circulado en el contexto reduccional. Pero también en este campo de investigación hay documentos hasta hoy no publicados, que nos pueden ayudar a ampliar nuestra mirada sobre estas cuestiones. Uno de estos documentos consta en la Wellcome Library de Londres, una de las colecciones más importantes de documentos de historia de la medicina. Se trata del manuscrito de Marcos Vilodas, hermano de la Companía de Jesús, llamado hoy Pohã Ñanã. Fechado en 1725, el texto permanece hasta hoy inédito. En lo relativo a recetas medicinales y terminología de plantas, el manuscrito constituye un aporte mayor a la medicina jesuita en aplicación en las Reducciones. A diferencia del Materia médica misionera, en el caso de Vilodas tenemos un texto escrito completamente en guaraní. A este panorama se tiene que considerar también una versión -también descubierta por Obermeierconservada junto con un texto de Gregorio López, o por lo menos circulando con el nombre de ese autor. Uno de los manuscritos de ese texto, escrito en México, consta en la Biblioteca Nacional de Madrid bajo el nombre de Libro de medicina en Lengua 
Guaraní, como parte de una colección de manuscritos de López y Vilodas, que en el catálogo sólo identifica a Gregorio López como autor. Todavía no hemos tenido la oportunidad de examinar a fondo estos materiales, pero lo más probable es que la parte en guaraní, escrita en las reducciones por un jesuita o médico, sea una versión desconocida hasta hoy de Marcos Villodas, diferente del manuscrito de la Wellcome Library. Esa versión fue añadida al conjunto en el momento de la encuadernación, porque sabemos que los textos de Gregorio López circulaban en varias reducciones jesuíticas. El examen de estos textos puede darnos a conocer no solo aspectos de la medicina colonial, sino también terminología etnobotánica en guaraní que quizás no consta en otros documentos, religiosos o historiográficos. Esto nos va a dar, dentro de este proyecto PeKY, en una segunda etapa de trabajo, un material o base de datos para un diccionario del guaraní jesuítico correspondiente al siglo XVIII, con citas concretas de los documentos.

\section{Nuevos aportes de la investigación lingüística}

Desde el punto de vista lingüístico, el valor de estos documentos es extraordinario. En primer lugar porque permiten una visión más general del complejo dialectológico del guaraní reduccional, conocido hasta ahora casi únicamente a través del corpus de escritos religiosos y de la obra lingüística de Ruiz de Montoya (Cf. Melià 1992: 112 y ss; Melià 1969 [2003]: 323). Los manuscritos que presentamos arriba, referidos a la vida secular, complementan aquel conjunto mejor conocido y permiten abrir preguntas referidas a las convergencias y divergencias entre el guaraní "cristiano" (que los jesuitas crearon por transformación del guaraní tradicional de los indígenas) y el guaraní coloquial efectivamente hablado entre los indígenas reducidos en el siglo XVIII. Una comparación entre los textos de lo "eterno" y de "lo temporal” ¿nos permitirán confirmar la idea de la existencia de "dos guaraníes" en las Misiones del Paraguay, de un guaraní "relativamente pobre [en los campos semánticos ajenos a la religión] aprendido como lengua secundaria por los padres [...], y de un guaraní muy rico, transformado solo en parte por los jesuitas, hablado por los indígenas de las Reducciones"? (Thun 2008b: 169). En segundo lugar, este conjunto de documentos posibilitaría el estudio diacrónico del guaraní reduccional, en el sentido de que los documentos escritos por indígenas, a lo largo de más de un siglo de escrituralidad vernácula, podrían estar poniendo en uso normas desviantes de la variedad estándar normatizada en las escuelas jesuíticas. No obstante estas cuestiones, de interés eminentemente lingüístico, nos preguntamos ahora sobre el modo en que nuestra disciplina puede contribuir a la investigación histórica y antropológica de la sociedad reduccional. La corriente sociolingüística conocida como etnografía de la comunicación presta un marco adecuado para realizar esta tarea (Hymes 1974, cf. también Duranti 2009). En este marco tiene interés la pregunta por el significado de estos textos en el contexto en el que se originaron. Para responder esta pregunta, y siguiendo las hipótesis presentadas arriba debemos hacer una revisión de la historia reduccional en lo que hace a la formación y difusión de sus lenguas y variedades lingüísticas, al uso convencionalizado de estas para diferentes géneros 
discursivos, a su materialización en diferentes canales (oral/escrito), a los sistemas de valores que estos usos evocan, y a la existencia de relaciones sistemáticas entre los usos del lenguaje y la estructura social reduccional.

\title{
2.1. EI siglo XVII: la formación de una comunidad de habla
}

En el siglo XVII, la relación entre los jesuitas y el guaraní está inserta en el contexto de una política lingüística ya definida por el Concilio de Lima de 1583/1584-y repetida por el Sínodo de Asunción de 1603- que indicaba la necesidad de evangelizar a los indios en sus lenguas. En términos prácticos, esto significaba codificar y predicar la religión católica en guaraní, una "lengua general" que -como el quechua y el aymara en el Perú- abarcaba una mayor cantidad de población y se extendía también sobre poblaciones hablantes no nativas. Siendo el objetivo principal de los jesuitas doble, la "salvación de las almas" y la organización civil de los indígenas, su trabajo se dirige a sentar las bases lingüísticas de la vida cotidiana en el contexto de la evangelización. Los indígenas en este proyecto cuentan como poseedores de un conjunto de saberes tradicionales -entre ellos la lengua- que hay que transformar, con vistas a su adaptación a las nuevas pautas de vida cristiana y civil. En una cita de La Conquista Espiritual del Paraguay, leemos a un cacique indígena dando una visión particular de este proceso:

\begin{abstract}
"La libertad antigua veo que se pierde, de discurrir por valles y por selvas, porque estos sacerdotes extranjeros nos hacinan a pueblos, no para nuestro bien, sino para que oigamos la doctrina tan opuesta a los ritos y costumbres de nuestros antepasados" Montoya 1639 [1989]: 225
\end{abstract}

La actividad de los religiosos es descripta a partir de dos rasgos significativos: "hacinar" en pueblos y "hacer oír" (= "para que oigamos") la doctrina. Tanto la doctrina católica como los pueblos constituyen elementos nuevos en la cultura y cosmovisión tradicional. La doctrina que el indio escucha cuenta con dos características centrales: el tema es religioso, y la lengua se presenta como una "variedad general", no siempre ni necesariamente coincidente con la especificidad dialectal de la parcialidad indígena donde la prédica se produce. En efecto, la doctrina (catecismo y oraciones) había sido redactada en guaraní, posiblemente a través de los catecismos y artes ya disponibles de Bolaños y Roque González, pero está construida en vistas a cierta uniformidad o generalización, propios de la cultura escrita y cuyo objetivo prioritario fue el hacer inteligibles las verdades del cristianismo católico a un número potencialmente grande de gente indígena. Este hecho, fundado en la función referencial del lenguaje, dejaba afuera otros problemas de tipo contextual: los problemas de la pragmática del discurso (es decir, reglas para el uso social del habla) y los dialectalismos o variantes locales propias de toda lengua general. A partir de la experiencia previa en otras regiones, los religiosos sabían que una terminología heterogénea en los temas de la religión podría afectar la veracidad del discurso cristiano (Melià 1969 [2003]: 44-45, 68). Los primeros jesuitas 
que vinieron del Brasil tenían experiencia en el proceso de normalizacion lingüística, efectuado en su caso sobre un dialecto tupí para la formación de una "língua geral" de la costa atlántica ${ }^{2}$. En las Reducciones del Paraguay, que ocuparon hasta casi mediados del siglo XVII un espacio geográfico bastante amplio (desde el Itatín hasta el Guayrá y el Tape), las gramáticas y doctrinas que se escribieron fueron pensadas con un sentido unificador, pues debian servir para la Misión en general (Cf. Melia 1992: 28-29, 93). La obra lingüística de Ruiz de Montoya, el Tesoro de la lengua guaraní (1639) y el Arte $y$ vocabulario (1640), sintetiza casi 50 años de trabajo previo y constituye la cúspide de este trabajo de generalización lingüística en donde ya poco puede restituirse de los dialectos que sirivieron de base a la descripción (Cf. Melià 1992: 28-29 y Melià 1969 [2003]: 107 y ss. para esta problemática). La citada obra de Montoya, por lo demas, sirvió de modelo para toda la generación posterior de misioneros: "una vez impresa la gramática de Montoya, se constituyó en un libro de texto con el cual aprendieron la lengua los numerosos jesuitas que, de todas las naciones de Europa, venían a trabajar en las Reducciones" (Melià 1992: 86).

Si el rasgo sociolingüístico saliente de la misión jesuítica fue el manejo y difusión de una variedad general del guaraní, el rasgo más especificamente lingüístico fue la transformación de la lengua indígena en vistas a la trasmisión del dogma católico y los principios de la vida cristiana. Esta transformación involucró en gran medida el plano léxico y trajo aparejada la acuñación de términos nuevos mediante el calco semántico (tupâo, 'casa de Dios', 'iglesia', tupâsi i 'madre de Dios'), la invención directa (añareta 'lugar del diablo' 'infierno', tecomboyoyaha 'manera de hacer igual', 'justicia'), la modificación de acepciones tradicionales (tupa 'personificación de la divinidad' $\rightarrow$ 'Dios de los cristianos', paye 'médico, sacerdote' $\rightarrow$ 'hechicero malo', ĭbaga 'cielo' $\rightarrow$ 'cielo, paraíso', yecoacu 'couvade, práctica tradicional' $\rightarrow$ 'ayuno') (cf. Thun 2008b: 144 y ss). Evidentemente la importación directa de términos del romance no pudo evitarse, habiéndose introducido conceptos como purgatorio, Espiritu Santo, obispo, christiano, y otros, si bien todo indica que los jesuitas buscaron siempre proteger, en la medida de lo posible, al guaraní de la influencia del español ${ }^{3}$. Así, los jesuitas no cambiaron la lengua de los indígenas, pero crearon, aprendieron y difundieron una variedad que con el tiempo se constituía en una nueva tradición idiomática dentro del guaraní, tradición que, por su énfasis en asuntos de religión, puede llamarse con justeza "guaraní cristiano". Por su aspecto comunicativo, además, este "guaraní cristiano"

\footnotetext{
${ }^{2}$ Dialecto que fue usado más tarde para la misión en otros pueblos, tupíes y no tupies, por ejemplo en Amazonia. El trabajo gramatical efectuado por José de Anchieta (1595) sobre el dialecto tupí del litoral hablado por los tupinambá constituyó además un antecedente y un modelo para el trabajo que realizarían después los jesuitas del Paraguay.

${ }^{3}$ Véase el conocido pasaje del padre cardiel Cardiel, que quiere expresar el orgullo que sentían los jesuitas hacia su guaraní, llamado aquí «la propia [lengua] guaraní», por contraste al guaraní ya mixto del entorno colonial (cit. en Melià 1992: 59; Thun 2008a: 218)

${ }^{4}$ La transformación de la lengua, que no llega a modificar el tipo lingüístico nativo ni sus estructuras gramaticales, consiste sobre todo en la ampliación de posibildades combinatorias a nivel léxico (formación de palabras, p.ej. derivación de sustantivos abstractos con - haß que posee un alcance semántico bastante amplio: 'instrumento, modo, causa, intento, fin, tiempo, lugar...', Montoya 1639 [2011]: 138), en la
} 
se deja concebir como una variedad diafásica, un estilo o forma de hablar altamente especialidada en los géneros característicos de la prédica religiosa (el sermón, la oración, la confesión, el catecismo) y sus contextos altamente ritualizados.

Si esta es la variedad lingüística que hablaron los religiosos (e indujeron a hablar a los indígenas), muy a nuestro alcance por su vasta documentacion en la obra escrita por los jesuitas, poco sabemos de la lengua que usaron los indigenas reducidos del siglo XVII, sobre todo fuera de los asuntos de la religión. La guaranística actual supone razonablemente un proceso de koineización de los dialectos de los indígenas reducidos, proceso al que contribuyeron las migraciones, los reagrupamientos, la movilizacion masiva y relocalizacion de los pueblos jesuitas antes, durante y después del conflicto con los bandeirantes del Brasil (década de 1630). Estos procesos, junto con cierta uniformidad cultural de los pueblos de indios, habrían conducido al desarrollo de formas de hablar propias del entorno reduccional (Melià 1969 [2003]: 106; Melià 1992: 95-96, 156). Dentro de estas formas de hablar cabría diferenciar, en nuestra opinión, un guaraní "cristiano", monopolizado y controlado por los jesuitas, y un guaraní de la vida práctica, ligado a las tareas que desarrollaron los indígenas (cría de ganados, la agricultura, la producción manual, la administración), tareas en las que el purismo lingüístico debió importar poco frente a la practicidad comunicativa en esos contextos menos formalizados. Este guaraní de la vida práctica se habría especializado en un nivel coloquial entre los indígenas del mismo modo que el guaraní cristiano servía a funciones destacadas. Uno de los rasgos que convencionalmente se atribuye al guaraní no religioso de las Misiones Jesuíticas -y de los otros pueblos de indios no regenteados por los jesuitas- es el de un volumen mayor de hispanismos léxicos (Melià 1992: 32-34; Thun 2008b: 157 y ss). En campos semánticos "seculares" se constata con más frecuencia la presencia de préstamos ${ }^{5}$ : animales domésticos (cabayu, buro 'burro', ndovi 'novillo'), plantas (açafran, ceboya), herramientas (alicate, bari 'barril', cepinyo 'cepillo', navaja, siya), sustancias y metales (açupre, cabô 'carbón', promo 'plomo'), etc. El "guaraní cristiano", más a salvo de una hispanización por el celo religioso y lingüístico de los jesuitas, configuró por su parte una variedad normatizada, elevada a técnica escrita y estandarizada, y debió ser una suerte de "variedad alta" u oficial de las reducciones. Esta situación, de la que dan cuenta las gramáticas del siglo XVIII,

creación de neologismos y en la subsecuente reorganización de campos semánticos tradicionales, así como en el desarrollo de algunas estructuras inexistentes en la lengua indígena (para expresión de tiempos verbales, voz pasiva, etc.). Algunas oposiciones (por ejemplo elementos del habla masculina/ habla femenina) fueron simplificadas, generalizándose un uso en detrimento de otros. Habiendo evitado la entrada de préstamos directos del espanol en el guaraní cristiano (p.ej. protegiendo el contacto de los indios con el mundo colonial) los jesuitas no pudieron evitar cierta hispanización a nivel estructural, procedente de su propio manejo del guaraní como lengua extranjera (cf. Melià 1969 [2003]: 183 y ss., Thun 2008b: 144 y ss.)

${ }^{5}$ Se trata en parte de préstamos indirectos, de hispanismos y lusitanismos tomados de las ,lenguas generales“ -guaraní y tupí criollos- habladas en el entorno colonial, lenguas con un desarrollo anterior y paralelo al guaraní reduccional. Esas lenguas estaban ya caracterizadas por una mayor presencia de elementos alóglotas que servían para denominar objetos, prácticas y técnicas importadas de Europa, y que se habrían retransmitido, a su vez, al guaraní reduccional. 
como se verá, nos permite formular hipotesis sobre cierto desfasaje entre el guaraní de los jesuitas y el de los indigenas reducidos, y cierta especificación funcional de ambas variedades.

\subsection{Siglo XVIII: hacia una reorganización de los recursos lingüísticos}

Diversos elementos muestran que hacia principios del siglo XVIII tiene lugar una nueva fase en la valoración y organización del habla dentro de las Reducciones. En esta nueva fase se apoya en tres factores: i) el descubrimiento por parte de los jesuitas de que la "variedad cristiana" de guaraní que ellos aprendían y fomentaban aparejaba grandes limitaciones funcionales en la comunidad ii) el ascenso y valoración de formas de hablar procedentes de los indígenas alfabetizados que, en su ejemplo paradigmático, Nicolás Yapuguay, llegarán a desarrollar un género literario hasta ahora privativo de los religiosos: el sermón, iii) el incremento y la organización de la producción escrita en las Reducciones, que refleja una nueva fase en el trabajo de los jesuitas sobre el guaraní. Nuestra hipótesis es que el trabajo lingüístico de los jesuitas -grupo dominante, supervisor y a cargo de una "planificación lingüística" de las Reduccioneses movilizado en este período por una nueva valoración del habla indígena y por una conciencia mayor de sus variedades.

\subsubsection{Los jesuitas y el guaraní del siglo XVIII}

Entre los jesuitas el conocimiento de la lengua nativa fue siempre prioritario. Debían no sólo conocer la lengua, sino se esperaba de ellos que la hablaran con soltura y "eminencia". No obstante este requerimiento, la proficiencia de los jesuitas en guaraní fue desigual durante todo el tiempo de las Reducciones, existiendo entre los religiosos diferentes grados de competencia lingüística y distinguiéndose al que poseía mejor destreza con el calificativo de "lengua" o "lenguaraz". Hacia finales del siglo XVII y comienzos del XVIII, en un apretado tercio de siglo, hacen su aparición un número importante de tratados de lingüística: el vocabulario manuscrito Phrases Selectas ${ }^{7}$, de 1687, las gramáticas manuscritas de 1696 y de 1718 (editada en 1890), el Arte impreso

\footnotetext{
${ }^{6}$ En la segunda instrucción del padre Diego de Torres, de 1610, leemos "A ésta [la lengua] se atienda con sumo cuidado siempre, no se contentando con saberla como quiera, sino con eminencia: persuadiéndose que depende en segundo lugar de ello la conversión de los indios [...]" (cit. en Melià 1969 [2003]: 93)

${ }^{7}$ Algunos de los problemas de autoría y fecha del manuscrito Phrases Selectas, así como el estado actual de la investigación sobre este texto, nos muestra cierta visión parcial que tienen algunos especialistas sobre la lingüística jesuítica posterior a Montoya. Phrases Selectas está fechado en 1687, sin datos de autor. En esa fecha Restivo todavía no estaba en las Reducciones. Melià resuelve la cuestión concluyendo que hay un error en la data del texto, y que el autor es indudablemente Restivo (Melià 1992: 93). Para nosotros esa visión es discutible, así como la equiparación del pseudónimo "Blas Pretorio" con la persona de Restivo. A nuestro modo de ver existe en la investigación del guaraní jesuítico la tendencia de descuidar el aporte de varios jesuitas anónimos dentro de una obra tan vasta como la lingüística del guaraní reduccional. Esta tendencia también se expresa en una visión unilateral del guaraní reduccional, creada a partir de la focalización en la obra lingüística de Montoya. Esta obra ha servido para elaborar la idea de un "guaraní clásico", unificado y estandarizado, descuidando los fenómenos de variación bien documentados en manuscritos hasta hoy no editados como las Phrases Selectas.
} 
en las doctrinas de 1724, y el Vocabulario de 1722, agrupados en torno a la figura de Paulo Restivo y sus colaboradores. El denominador común de estos textos es reforzar el entrenamiento y práctica de los religiosos en la lengua indígena y de preparar a los nuevos misioneros en el conocimiento del idioma. Esta intención, que es también visible en las primeras obras de lingüística misionera, desde Bolaños en adelante, viene ahora relacionada con un factor distintivo: la nueva producción busca "actualizar" los trabajos de Montoya, trabajos que hasta ahora constituían la norma del habla jesuítica. El desfasaje entre la "variedad cristiana" y las hablas de los indígenas se pone así en descubierto. En Phrases Selectas, el énfasis es puesto en la variación diacrónica y diatópica:

\begin{abstract}
"Con mucha razón llamó Tesoro el venerable padre Antonio Ruiz al que compuso sobre el idioma guaraní, para los principiantes muy escondido, ya por los yerros de la imprenta, ya también por algunos vocablos, y modos de hablar, que aunque en la realidad son vocablos, y términos propios de los naturales, pero ya per non usum se han antiquado y hecho casi inteligibles [...] No digo esto, (benévolo lector) sin alguna experiencia, porque en San Javier se usan modos de hablar tan particulares, que valiéndome yo de ellos en Santa María y en otros pueblos, no me entendían, y fue necesario mudar de rumbo, y buscar otros usuales en aquel pueblo [...] también porque leyendo algunos párrafos [del Tesoro] a los naturales no me entendían" Phrases Selectas, Introduccion sin firma de autor.
\end{abstract}

La cita muestra que existe una preocupación por la situación lingüística de la comunidad, en donde el saber idiomático de los jesuitas no es suficiente para hacerse entender en diferentes contextos. Por otra parte, los numerosos ejemplos y observaciones del Phrases Selectas, así como de otros textos arriba citados, indican que la preocupacíon de los jesuitas no se centra sólo en el estilo "alto" de la prédica religiosa, sino que se extiende también (y por primera vez) a los registros lingüísticos de la vida social ajena a lo religioso ${ }^{8}$.

\footnotetext{
${ }^{8}$ Cf. algunas observaciones de Restivo. Con respecto a cómo los indígenas usan el verbo aypea apartar (algo)": "aypea es activo que dice 'lo aparto' [...]. Nota que también dicen: ahaühucañy, ayeaĭhucañ̃y siendo assi, que el cañy ['perder'] es verbo absoluto [...]. La razon será porque quando el indio dice ahaĭhu cañy quiere decir: no ay mas en mí, se acabo el amor que yo le tenia, que equivale al otro" (Restivo, Linguae Guarani, 1724 [1892]: 328) Otras veces, el autor destaca los usos más aceptados: "Estas oraciones se pueden hazer también de esta otra manera: ...; pero el primer modo [...] es mejor y mas usado" (íbid, 187) "Los modos de hablar siguientes son muy enfáticos y [...] son modos muy usados en las composiciones de Indios y Padres lenguaraces" (íbid, 194). Los comentarios de orden pragmático son también numerosos: "Nota que quando digo 'reprender' no se ha de tomar por reprehension rigurosa, pues también se puede usar aun hablando con persona de respecto" (íbid, 200). Véase también Melià 1969 [2003]: 179-180.
} 


\subsubsection{El ascenso de una elite letrada}

Los grandes géneros retóricos que focalizaron la atención de los jesuitas durante el siglo XVII fueron el sermón y otras extensiones de la práctica evangelizadora (catecismo, confesionario, oraciones). A este género y a esta intencionalidad se habían subordinado la apropiación de la lengua y la producción de escritos y guías gramaticales del siglo anterior. Hacia el siglo XVIII este panorama se habría modificado, ya que los indígenas comienzan a dominar a su vez estos géneros, no sólo de manera oral, sino también en lo escrito. Se trata, básicamente, de los indígenas alfabetizados. El siguiente fragmento de Peramás testimonia varios elementos de esta nueva situación que podemos llamar los "efectos" de la alfabetización:

"Como los guaraníes no entendieron los libros castellanos, pusiéronse los jesuitas a escribir libros en guaraní y al efecto compusieron muchísimos, no poco de los cuales fueron impresos. Los indios los leían con avidez. Pero no sólo leían esos libros, sino que ellos mismos escribieron otros. Yo he visto y semanalmente he leído los discursos que un indio de Loreto escribió sobre los evangelios de diversas domínicas, tan elegantes que ningún jesuita había podido componerlos mejor. Y ese indio escribió su libro en esta forma: cada domingo prestó atención a lo que el párraco explicaba desde el púlpito, y los comentarios que hacía, meditaba después lo oído,y, pluma en mano, reproducía en purísimo guaraní, los conceptos e ideas, agregando de su cocecha lo que creía más adecuado y oportuno. De esta manera llegó a componer un libro que prestó grandes servicios a todos los misioneros, y del que yo me valí muchísimo para mis sermones" Peramás, 1768 [1952]: 594.

A partir de esta cita $-\mathrm{y}$ de otras que replican en líneas generales esta información' ${ }^{9}$ destacamos tres aspectos relevantes. En primer lugar, el proceso de comunicación escrita cuenta ahora con un nuevo tipo de participante, tanto en la lectura directa como en la escritura: el indígena que, perteneciente a un subgrupo especial de las reducciones, había asistido a las escuelas que los jesuitas impulsaron desde el principio de la Misión. La escritura fue promovida sólo entre algunos caciques y su descendencia, y excepcionalmente en niños con destrezas especiales, con lo que se habría formado una suerte de "elite" letrada que iba a desempeñar cargos en los cabildos y en la administración de las estancias (Melià 1992: 137, Neumann 2007:

\footnotetext{
${ }^{9}$ Sigue Peramás: "Autores de libros no religiosos, conocí yo a dos indios. Uno que se llamaba Melchor y escribió la Historia del pueblo de Corpus Christi. [...] Otro libro fue escrito por un indio del pueblo de San Javier [...] Refería cómo los jesuitas habían llegado a sus tierras, cómo fueron recibidos por sus antepasados, relata la muerte del Venerable Padre Roque González [...]" 1768[1952]: 594 y ss. También existiría una crónica en guaraní del pueblo de Yapeyú (ver Melià 1992: 141).
} 
Cerno - Obermeier. Lingüística e investigación de documentos en guaraní (siglo XVIII).

51). Los indígenas de esta casta, que hasta mediados del siglo XVII parecen haber participado del proceso de escritura sólo como copistas o escribas, pueden ahora escribir por su cuenta y al parecer, con relativa autonomía, como sabemos según la explicación que Peramás da sobre el "método" que sigue el indio aludido. En segundo lugar, los halagos que merece en la cita la prosa indígena (calificada de "elegante" y de escrita en "purísimo guaraní") dan una pista acerca de una clara diferenciación sociolectal (“purísimo guaraní") y también estilística (“elegante”) entre los sermones compuestos por los jesuitas y por los indígenas. Ambas cualidades son valoradas por su utilidad, pues sirven para que los jesuitas compongan sermones acaso más efectivos imitando el "estilo" del indígena ${ }^{10}$. Debe llamarse la atención de que un estilo es algo más que una forma de organizar los medios expresivos para ciertos fines retóricos: su efecto depende del reconocimiento de formas de hablar provistas por ciertos estratos o sectores de la comunidad (cf. Hymes 1974). Si seguimos esta pista ¿podemos plantear que, después de un siglo de cohesión sociocultural, en las reducciones el "guaraní cristiano" de los jesuitas es manejado ya, con mayor espontaneidad, riqueza y efectividad, por los hablantes nativos, particularmente por los integrantes de esta casta escolarizada que además es capaz de escribir sobre los asuntos "altos" de la religión? Una comparación entre el estilo de Yapuguay y el de los jesuitas escritores de sermones, todavía por hacerse, podría aproximarse a una respuesta. En tercer lugar, si los indios letrados son capaces de dominar los géneros retóricos "de lo eterno", este hecho quitaba a los jesuitas el monopolio que habían ejercido hasta ahora sobre el "guaraní cristiano" con el que hablaban de religión. La reacción de los jesuitas habría sido práctica: como sugiere Peramás en la cita, la competencia del indígena se constituye como un modelo, pero debe "prestar servicio" a los misioneros. La relación entre Restivo y Nicolás Yapuguay parece ejemplar en este sentido: "Dicho padre [Restivo] tuvo siempre a su lado a este indio [Yapuguay] y le consultaba siempre que quería expresarse con más elegancia en el idioma guaraní" (Peramás 1768 [1952]: 594., cit. en Melià 1992: 140). Restivo consulta a Yapuguay, lo cita, lo imita, lo erige como modelo, pero esta relación no es simétrica: el indio escribe sus sermones "con dirección" de un padre de la companía de Jesús (cf. Thun 2003: 13), encuadrándose los escritos de Yapuguay en el período de escrituralidad "controlada" referido más arriba. La creacion de una clase indigena de mayordomos y secretarios de la administracion reduccional, por lo demás, no habría influido sólo en la renovación del guaraní religioso, sino también en cierta normalización de un guaraní práctico: la alfabetización habría creado un grupo de personas que poco a poco empieza a utilizar la escritura para fines cotidianos: cartas a otras reducciones, "billetes", recibos, actas de cabildos, etc. Esa clase letrada muestra

\footnotetext{
${ }^{10}$ Un comentario de Restivo sobre el método de su Arte de ...: "me puse de propósito á juntar en uno [libro] lo más selecto que en cada uno dellos [autores jesuitas de Artes] he hallado [...] que es esta obrita, que te ofrezco, en la qual he tambien añadido muchas otras Anotaciones y reglas, que yo he sacado de varias composiciones de Indios y del P. Simón Bandini, tenido comunmente por Príncipe desta lengua, aviéndolas primero averiguado con Indios muy capaces [...]" Restivo, Linguae Guarani, 1724 [1892], "Al Lector", p. 5, el destacado es nuestro.
} 
de este modo a los jesuitas otra variedad de la misma lengua, acompañada ademas del prestigio que le habría conferido la escritura.

\subsubsection{La organización de producción textual entre 1687 y 1733}

A continuación examinamos la producción escrita, anterior, contemporánea y posterior a la primera imprenta jesuita (cerca de 1705-1727), en vistas a una ilustración de las ideas expuestas arriba y de una interpretación de los hechos más relevantes en torno al uso escrito del guaraní en esta etapa. La imprenta -y el eventual "scriptorium" de preparación de manuscritos- constituye un escenario fundamental, único en el Río de la Plata y culturalmente específico de las Reducciones. En este escenario se concentra el trabajo de jesuitas lingüistas, que ejercen la actividad de la traducción, y de indígenas competentes en la elaboración de textos en guaraní. El período aludido es significativo además porque se trata de una fase de gran producción textual, donde debemos contar tanto la obra metalingüística centrada en torno a la figura de Restivo (§ 2.2.1), como el conjunto de manuscritos presentados arriba ( $§ 1)$, y el vasto cuerpo de producción textual manuscrita e impresa que va desde De la diferencia entre lo temporal y lo eterno (1705) de Juan Eusebio Nieremberg, hasta las traducciones de la Conquista espiritual al guaraní (1733) (ver Furlong 1955; Melià 1992: 126 y ss.). Examinaremos por separado algunas variables que creemos pertinentes en términos de un análisis global de esta producción textual. Tomamos para esto por separado las decisiones de los jesuitas en torno a la selección del canal de los textos (impreso/ manuscrito), los géneros discursivos textualizados, y la variedad dialectal o registro que será llevado a la escritura.

Con respecto al soporte textual, no debe pensarse en términos contemporáneos suponiendo que el manuscrito posee un valor inferior al impreso y constituye una alternativa "no oficial" de la circulación de lo escrito. La imprenta jesuítica es de hecho la primera imprenta del Río de la Plata y los jesuitas debían considerar el impreso como una posibilidad de mayor difusión, siendo la variante más normal el manuscrito, que había orientado la producción textual interna de las Reducciones hasta entonces. Sin dudas el impreso constituye, para la época, una jerarquía superior, más efectiva para dar prestigio al trabajo propio: la traducción de Nieremberg, impresa, está dedicada al General de Roma Tirso González de Santalla. La imprenta también constituye una manera de mostrar que la Orden es capaz de manejar la lengua indígena no sólo para la catequesis, sino también para una alta terminología teológica como la de Nieremberg.

Con respecto a los géneros discursivos desarrollados, tenemos en esta etapa una diversificación hasta ahora no conocida en la literatura oficial del contexto reduccional, donde debemos contar con tres formas: dentro del género religioso, debemos discriminar entre i) una literatura ascético-filosófica y ii) las obras de carácter más práctico que debían servir a la elaboración de la prédica, y que son los catecismos y sermonarios. Este conjunto religioso se complementa con iii) una literatura de carácter más profano, centrada en el mundo secular. Entre los textos de alta teología e ideas morales 
abstractas contamos el ya citado De la diferencia entre lo temporal y lo eterno (1705) de Nieremberg, primer libro impreso en el Río de la Plata, al que nos referiremos más adelante. Las obras de carácter práctico (en el campo religioso) son el Maryrologium Romanum (de conservación fragmentaria pero probablemente anterior al Nieremberg, impreso en torno de 1700, ver Maeder 2008), calendario religioso con los mártires más importantes de la Iglesia, el Flos Sanctorum, perdido actualmente, presuntamente impreso, y que sigue esta misma línea práctica; la colección de Catecismos Varios (1716), manuscritos inéditos hechos para la prédica y explicación de la doctrina cristiana, conservados actualmente en el British Museum (edición por Plínio Ayrosa 1952-1956); el Manuale ad usum Patrum (1721, ver Pallomera Serreinat 2002, con traducción de las partes en guaraní), libro que debió servir para los oficios religiosos, y finalmente las obras Explicacion de el Catechismo (1724) y los Sermones y Exemplos (1727) de Nicolás Yapuguay, impresos que cierran la época de la imprenta reduccional. Entre los textos más próximos a la vida secular se cuentan principalmente obras de género historiográfico. La historiografía, género importante para los jesuitas, contó con un desarrollo cuyo ejemplo más conocido son los varios manuscritos de La conquista espiritual del Paraguay (1733) traducida al guaraní. Aquí debemos destacar también el caso de otro manuscrito, recientemente hallado, que parece ser la traducción al guaraní de la Historia de Nicolás del Techo ${ }^{11}$ sin fecha, aunque posiblemente elaborado en este mismo contexto. Hoy podemos estar más seguros del desarrollo del género de la crónica histórica con la aparición del Diario de un desalojo (1704-1705) citado y descripto más arriba (§ 1.2.). Otros géneros de temática no religiosa son los diálogos del Manuscrito Gülich (sin fecha, probablemente contemporáneo a estos textos) y el texto de farmacología y medicina Pohã Ñanã (1725).

Con respecto a la lengua y las variedades impresas, es evidente que la elección del guaraní se corresponde con la necesidad de producir materiales para "uso interno", sobre todo de los religiosos (preparación de sermones, incremento de la competencia lingüística), pero sin descuidar la lectura de los indígenas letrados. Con respecto al registro, la lengua debe adecuarse no sólo a la competencia de los religiosos, sino, y sobre todo, al saber idiomático de los indios. En uno de los paratextos de la primera obra publicada en la imprenta jesuítica, De la diferencia entre lo temporal y lo eterno (1705) de Juan Eusebio Nieremberg, leemos que el libro:

"Se debe imprimir. El estilo está claro, inteligible, muy natural y ajustado a las reglas del arte y uso común de los indios. [...] Consta haberse recibido con aprecio de los naturales las veces que han oído algo del dicho libro" Medina 1892, Historia y bibliografia de la imprenta en la América española, cit. en Melià 1992: 123 (ver también nota 10)

\footnotetext{
${ }^{11}$ Esta es al menos la primera interpretación que da a este manuscrito Franz Obermeier, coautor de este artículo y responsable del hallazgo.
} 
Después de un siglo de desarrollo y uso del guaraní cristiano, después de su difusión entre los propios indígenas, la aceptabilidad del discurso religioso parece depender menos de su función referencial (el qué se dice) que de las formas socialmente aceptables de hablar que despliega (el cómo se dice), formas cuyo modelo se identifica con el indígena. Si el trabajo de la escrituralidad jesuítica, hasta ahora, pareció haber estado gobernado por una "lógica de traducción" (Cf. Melià 1992: 119), en el contexto de la imprenta estamos arribando a una "lógica de imitación", posible sólo cuando el hablar de los indios se eleva a modelo.

Resta ahora una interpretación de estos datos a la luz de la obra metalingüística de los jesuitas en esta etapa. En primer lugar, debe pensarse que tanto el género religioso como el profano constituyen una producción contemporánea a la obra de Restivo y otros autores desconocidos (§ 2.1.1.). ¿Debemos atribuir esta concentración de la producción escrita a los azares de la conservación de manuscritos, o podemos brindar una explicación satisfactoria desde un punto de vista sociohistórico? Nuestra hipótesis es que existió un programa o estrategia general de los jesuitas en torno a la lengua de las reducciones hacia el siglo XVIII, y que esta estrategia buscó completar la obra comenzada en el siglo XVII.

Comenzamos con un análisis de las circunstancias y condiciones de publicación del primer impreso, el De la diferencia entre lo temporal y lo eterno de Nieremberg ${ }^{12}$. El hecho de que ya hubiera una traducción de Nieremberg al chiquito, lengua general de las Reducciones de Chiquitos ${ }^{13}$, nos hace pensar que su traducción al guaraní responde a una estrategia general de los jesuitas, entroncada con su concepción "lingüística" de la evangelización. Ya ha sido dicho que el objetivo de traducir el texto respondía a la doble necesidad de ofrecer material para mejorar la proficencia lingüística de los religiosos, y de ofrecer lectura a los indios. A este objetivo debe sumarse también la posibilidad de desarrollar la lengua indígena para la expresión de altas ideas teológicas y morales expresadas en esta obra (Cf. Melià 1992: 124). A diferencia del siglo XVII, donde la necesidad del momento derivó en la estrategia de elaborar gramáticas y diccionarios paralelamente a textos religiosos de orden práctico, el proyecto de traducir Nieremberg habría constituido el principio de una etapa nueva, donde debía evaluarse el alcance del guaraní para temas más elaborados. El Nieremberg pudo haber sido una suerte de "laboratorio" de experimentación lingüística para poner a prueba la lengua indígena y medir qué se podía hacer con ella a ese nivel. Este texto fue elegido para la traducción porque, lleno de "exemplos" con sentido moral, escrito por un jesuita muy

\footnotetext{
${ }^{12}$ Sobre los antecedentes de investigación acerca de este texto capital deben contarse apenas aproximaciones desde la historia de la imprenta en el Río de la Plata y desde la iconografía (Sebastián 1992, Obermeier 2006, Gil 2010), siendo inexistentes los aportes de la sociolingüística histórica (Cf. Melià 1992: 123). No hay trabajos sobre el lenguaje usado en este texto, $y$, hasta donde sabemos, tampoco sobre el lenguaje religioso en general fuera de la gran cantidad de investigación concentrada sobre Montoya (p.ej. casi toda la obra de Melià). Esto significa que nuestras observaciones a nivel lingüístico deben ser consideradas hipotéticas.

${ }^{13}$ Actualmente en el Archivo Parroquial de Santiago de Chiquitos, 32 páginas en el Archivo Histórico de Chiquitos, actualmente en Concepción, (donación Riester, $n^{\circ}$ 1), ver sobre esa tematica Falkinger 2008.
} 
popular en la época, podía ser utilizado como base en la elaboración de los sermones, ofreciendo además a los jesuitas modelos de expresión para ideas de gran nivel de abstracción. La contraparte de esta literatura teológica es el Martirologium Romanum ${ }^{14}$, casi contemporáneo al Nieremberg y caracterizado por su temática menos filosófica y su posibilidad de usarse mejor en la práctica cotidiana de la actividad religiosa. Si estos dos textos, de tipo religioso, se oponen entre sí por el alcance de su temática (teórica/ práctica) y por su probable utilización (traducción y desarrollo de la lengua/aplicación en el sermón) ¿podemos extender esta lógica al conjunto mayor de la producción escrita de esta fase? Nuestra idea es que este "laboratorio" de experimentación con la lengua concentró su actividad en la elaboración de todas las obras escritas en guaraní. Podemos pensar en una especie de "Scriptorium", cuyo trabajo dirigieron los jesuitas y ejecutaron los indígenas, y que habría acompañado la labor de la imprenta en Santa María Mayor o en Loreto. Este "Scriptorium" habría estado paralelamente centrado en aspectos de producción textual como en el código o registro adecuado para cada uno de ellos. Dado que la primera motivación de la obra metalingüística en esta fase, según se encuentra expreso en Phrases Selectas (1687), es el ajuste del habla de los religiosos a la variación de la lengua de los indígenas, se infiere de aquí que existió una necesidad de estandarización y la conciencia de tener en cuenta diferentes normas y subestándares. El hecho de que la obra metalingüística de Restivo, de "Blas Pretorio" y de los colaboradores anónimos coincida con la producción textual religiosa e historiográfica indica la idea de aplicar el conocimiento lingüístico-dialectológico y estilístico reciente a la práctica de la escrituralidad. De este modo los lingüistas jesuitas del siglo XVIII trabajaron en varios dominios: si bien la religión ocupó una posición de prestigio, indicada por el acceso a la imprenta de las obras religiosas y el mantenimiento de las obras "de lo temporal" en su carácter de manuscritos, los padres no dejaron de observar la funcionalidad de la lengua en campos ajenos a la religión. El hecho de la escritura de discurso histórico, medicinal y de diálogos de la cotidianeidad indica que todos estos textos habrían servido paralelamente para ampliar la competencia lingüística de los religiosos fuera de los asuntos de la religión, para extender las posibilidades de la lengua a temáticas todavía no desarrolladas, y para alcanzar una normalización lingüística deseada que pudiera fijar variedades "altas", "no marcadas" y "bajas" del guaraní, registros que sirvieran para hablar de asuntos serios, neutros o "bajos" (p.ej. el humor presente en el Manuscrito Gülich o el habla de los indígenas representados en el Diario de un desalojo) y cuyo modelo en el habla indígena había sido comprendido y aceptado. Los textos de temas no religiosos permanecieron en carácter de manuscritos, hecho que indica también la posibilidad de ser revisados y corregidos, o considerados todavía provisionales. Por otra parte, el hecho de que varios catecismos y sermones hayan circulado también en forma manuscrita (p.ej. el Sermones Varios), y que una versión haya alcanzado la forma impresa en la obra de Nicolás Yapuguay recién en 1724 sugiere que el trabajo con los sermones no había todavía satisfecho los criterios

${ }^{14}$ Obra calcada sobre el modelo latino y reformada por el jesuita Dionisio Vázquez en la edicion de Madrid, 1661, o en la posterior de Barcelona, 1700 
de evaluación, y que se llegó a requerir un modelo más válido surgido finalmente de la pluma del escritor indígena.

\section{3. ¿Un "guaraní cristiano" y un "estándar coloquial reduccional”?}

Veamos ahora qué aspecto tendría este guaraní centrado en lo cotidiano y qué preguntas y temas de interés se suscitan en torno a él. Para ello hacemos una aproximación a un fragmento del texto Conserva apo reco rehe Tembiu apoha ñomongetahaba tenicobaè "Donde se habla de recetas para hacer conservas", extraido del Manuscrito Gülich:

1. P: Mbaè ybata tamo ñamoeê amo curiegua ararenonde raè hê? 'Qué frutas aderezamos para que puedan durar más tiempo?'

2. R: Cidra yepe amo ñamoê̂ curie rey 'Hasta sidra podemos aderezar'

3. P: Haèbe yepe aypobaè rehe ñande ñemboçacoỳboŷ biña; haè aete raco ndoico çapiay ete eguî y̆ba yroetebaè hê

'Por eso mismo nosotros las preparamos. Pero no se hace esto de prisa, pues son frutas muy amargas'

4. R: Ayporamo yayapo çandia, cotera arasay rehegua; âbaè ño raco acerânge ramo, yyaye raibi hê; bìte naco acoybaè mburucuya, haè aguaŷh hê

'Entonces hagamos de sandía o de guayaba; sólo ésto cuando se tiene prisa hay que hacerlo rápido. Aún con el mburucuya y con el aguaí'

5. P: Chemaênduà yepe acoibaè rehe; haè aete yayapepî yepe co sidra rânge tenonde hê, hà̀ yuquĭri pĭpe ymoî yacatu chayapo eguî yba yroeỹbaè ymombaboybo ñote âng hê

'hasta me acuerdo de eso; tenemos que abrir la sidra, en la salmuera la ponemos, pues, hacemos frutas no amargas para terminar rápidamente ahora'

6. R: Eguî rami haèbene rêy

De esa manera se hará

$$
[\cdots]
$$

7. P: Haè ymboyı̆ parireê acoy cidra chamboyaò asucar reheguarâ arire hê 'Después de cocida totalmente la sidra, las cortamos y rociamos con azucar'

8. R: Ney ndahaèbeŷn naco eguibaè mboyaò boŷymboyı̃ eymobe hê Bueno, ciertamente no se puede cortarlas antes de que se cuezan 
Este texto ejemplifica el lenguaje puesto en el contexto de una actividad social, en este caso la preparación de conservas de frutas. El formato textual es el diálogo, la persona gramatical más empleada es el "nosotros" inclusivo, reflejado por el prefijo activo ya- (4), (5), su variante libre cha- (5) (7), su variante obligatoria nasal -ña (1), (2), 3), y el pronombre ñande 'nosotros' (3). Una pregunta que surge, conociendo la gramática del guaraní, es si el texto podría estar ejemplificando el uso, difícil para un hablante extranjero, de esta primera persona del plural "inclusiva" (nosotros = yo + tú, $+/$ - el, ellos) como alternativa al uso de la primera persona "exclusiva" que esta lengua expresa mediante la oposición con los morfemas ro- y ore- $($ nosotros = yo + él, ellos tú, ustedes). Si esto es cierto ¿tendría este texto, además de una función representativa, también una función autorreferencial, expositiva del valor de ciertos usos en ciertos contextos típicos? Sería interesante un análisis más amplio para alumbrar cuestiones de este tipo que aquí no podemos más que plantear.

En su aspecto léxico, el texto presenta una mayor cantidad de hispanismos que los textos sagrados, lo que podría significar la existencia de una actitud diferente a la "lealtad lingüística" que los jesuitas mostraron siempre hacia el guaraní, que se traduce en una mayor tolerancia hacia usos desviantes en ciertos contextos. Un ejemplo es el uso de asucar en (7) como alternativa al neologismo eyripia 'azúcar', ya acuñado por los jesuitas -entre otros neologismos (Thun 2008: 159, 164). Nótese además que la palabra asucar se escribe regularmente de esa manera a lo largo de todo el diálogo, evitando otras posiblidades como açucá o asucá, hecho que ya sugiere una normatización. Otros hispanismos son conserva, sidra y çandia, pero se conservan las formas en guaraní para el léxico etnobotánico: arasay 'guayaba', mburucuya y aguay. ¿Representará este guaraní coloquial reduccional una variedad que más tarde influyó en las lenguas mixtas actuales del guaraní, es decir en las variantes del guaraní paraguayo y correntino? (cf. Cerno 2013: 33). En el aspecto pragmático, resta reseñar la ocurrencia de usos "nuevos", desviantes con respecto a la tradición registrada por Montoya. La partícula hê 'ey, mira' colocada al final de los enunciados (1), (3), (4), (5) y (8), cuenta con un significado modal-enfático. Su uso, como el de las partículas en general, sigue pautas idiomáticas difíciles de codificar en una gramática y muy ajenas a las posibilidades de los hablantes no nativos. Montoya y Restivo dan, por otra parte, sentidos diferentes para esta partícula: mientras el primero indica 'hola, mira' para hê (cf. Montoya 1639 [2011]: 159), Restivo agrega un componente semántico propio del habla masculina: "ola, dice el varón al varón” (Restivo 1722 [1893]: 258). ¿Habrían prestado los jesuitas, hacia el siglo XVIII, mayor interés a la diferencia entre habla masculina y habla femenina, no habiendo bastado la descripción del siglo anterior? ¿Sería esta diferencia más pertinente en el habla coloquial que en el habla religiosa?

La existencia de esta clase de textos nos muestra que la riqueza del lenguaje coloquial no pasó desapercebida a los jesuitas, que habrían comprendido la necesidad de transformar también su propia norma de aprendizaje y su saber idiomático del guaraní. Ya no bastó un registro erudito y religioso, sino que, para hacerse entender en 
el ámbito de una norma coloquial y en campos semánticos diversos, hubo la necesidad de saber expresarse en otros dominios temáticos.

\section{Conclusiones}

Nuestro estudio muestra que entre las décadas finales del siglo XVII y primeras del XVIII los jesuitas buscaron orientar su trabajo lingüístico hacia el dominio de aspectos que habían caído fuera de su foco: la variación interna del guaraní, el lenguaje coloquial y sus valores pragmáticos y estilísticos. Después de la creación de un lenguaje religioso y de herramientas verbales para la prédica, cumplidas en las primeras décadas de misión, el foco está puesto en la lengua que el indígena emplea en la cotidianeidad de las reducciones ya consolidadas como espacio sociopolítico, económico y religioso particular. En referencia a los nuevos documentos, estos formarían parte de una estrategia que perseguiría, a la vez, la estandarización de nuevas variedades para la escritura, y la preparación de los misioneros en la esfera del discurso, dimensión que complementa el dominio del léxico y la sintaxis. Parte de esta preparación habría consistido en la introducción y práctica de los religiosos en asuntos de lo "temporal", expresados ahora con una variedad que podía considerarse un "estándar coloquial reduccional", presumiblemente identificado con el guaraní de los indígenas letrados. Por otra parte, estos manuscritos abren también interrogantes para la lingüística, en el sentido de que se presenta en ellos la cuestión de las variedades indígenas más alejadas de los estándares jesuíticos y su documentación histórica. Este interrogante llevaría a plantear una posible diglosia entre las variedades del guaraní reduccional, así como adelantar en cuestiones de diacronía, sentando las bases documentales para el estudio de la evolución del guaraní colonial hasta su situación presente.

Nuestro estudio ilustra además el modo en que la lingüística puede contribuir al estudio histórico y antropológico de la sociedad reduccional. Varios de estos documentos, como el Gülich y el Diario del desalojo, describen en parte aspectos conflictivos de la vida social, y de aquí puede afirmarse que hubo entre los jesuitas una necesidad de considerar más de cerca ciertas prácticas y su dinámica propia, previendo una serie de problemas típicos como la infracción de normas y el modo de actuar frente a ello. Estos problemas fueron enfrentados en parte considerando su dimensión comunicativa, previendo la variedad lingüística y el estilo más conveniente para intervenir en ellos. En este sentido tales documentos estarían representando (y autorreferencializando) ciertos actos de habla que permiten dirigir la vida social y actuar en ella. Es tarea de la lingüística guaraní lograr un conocimiento más preciso de las variedades y registros comunicativos que sirven para construir estos actos de habla. Para los historiadores y antropólogos, el conocimiento de una pragmática lingüística de la sociedad reduccional podía servir para discutir con mejores datos las cuestiones relativas a la "perspectiva del indígena" o a una identidad cultural de los guaraníes, tal como esta se estaría manifestando en los diferentes usos del lenguaje. El presente estudio sienta las bases 
Cerno - Obermeier. Lingüística e investigación de documentos en guaraní (siglo XVIII).

para pensar estas cuestiones, sobre todo en lo relativo a la primera mitad del siglo XVIII, aunque también motivando la investigación lingüística de etapas posteriores.

\section{Referencias bibliográficas}

Anchieta, Josephde. 1595 [1999]. Arte de gramática da língoa mais usada na costa do Brasil. 2 T. Madrid, Ediciones de Cultura Hispánica, Agencia Española de Cooperación Internacional.

Ángelis, Pedro de. [hacia 1853] Colección de obras impresas y manuscritas que tratan principalmente del Río de la Plata, [En línea sin apéndice]: http://www.cervantesvirtual. com/. Para el Apéndice, cf. el ejemplar de la Unversidad de La Plata.

Anónimo. 1687. Phrases selectas y modos de hablar escogidos y usados en la lengua guaraní, sacados del Thesoro escondido que compuso el venerable Padre Antonio Ruiz de nuestra Compañía de Jesús para consuelo y alivio de los fervorosos misioneros, principiantes en la dicha lengua, 633 p, según la "Advertencia": fechada en San Francisco Javier, 27.09.1687, Museo Mitre, Buenos Aires, Nr. del catálogo 4327

Cerno, Leonardo. 2010. "Las partículas modales en guaraní jesuítico. Una aproximación al discurso de "lo temporal”". En: XIII Jornadas Internacionais sobre as Missões Jesuiticas, Universidad Federal da Grande Dourados, Agosto 30-Septiembre 03, 2010; Dourados, Brasil. Edición cd-rom.

Cerno, Leonardo 2013. El guaraní correntino: fonología, gramática, textos. Frankfurt am Main, Peter Lang.

Duranti. Alessandro. 2009. Linguistics Anthropology. A Reader. Oxford: Blackwell Publishing Falkinger, Sieglinde, 2008, "Gramáticas y vocabularios de la lengua chiquita / chiquitana", En: Primer Congreso Internacional Chiquitano, Universidad Técnica Particular de Loja Mayo 22-24, 2008; San Ignacio de Velasco, Bolivia. [En Línea] http://www.utpl. edu.ec/portalchiquitano/ images/stories/bibliotecas/archivo_interno/mision_chiquitos/ lamisionenamerica_sieglinde_falkinger_lengua_chiquitana_gramatica_vocabulario.pdf

Furlong Cardiff, Guillermo, 1953-1955, Historia y bibliografia de las primeras imprentas Rioplatenses 1700 - 1850.3 T, Buenos Aires, guarania [et alia].

Ganson, Barbara. 2003. The Guarani under Spanish Rule in the Río de la Plata. Stanford, Stanford University Press.

Gil, Fernando Miguel. 2010. "Introducción" en: Nieremberg, J. E., De la diferencia entre lo temporal y eterno, primera edición facsimilar en conmemoración al Bicentenario de la Revolución de Mayo, Instituto Bonaerense de Numismática y Antigüedades, Bolsa de Comercio de Buenos Aires, Bolsa de Comercio de Rosario. [Puesto en línea el 9 de agosto de 2010]: http://bibliotecadigital.uca.edu.ar/repositorio/investigacion/diferenciatemporal-eterno-juan-niere mberg.pdf

Hymes, Dell. 1974. "Ways of Speaking”. En: Duranti, Alessandro. Linguistics Anthropology. A Reader. Oxford, Blackwell Publishing, pp.158-171.

Maeder, Ernesto J.A., 2008, "Hallazgo en Bolivia del primer libro impreso en las misiones jesuíticas". En: Emprender en la Región, Revista de economia y negocios, Nr. 35, Corrientes, [En línea] http://issuu.com/emprender_en_la_region/docs/emprender_n35,

Melià, Bartomeu. 1969 [2003] La lengua guaraní en el Paraguay Colonial que contiene La creación de un lenguaje cristiano en las Reducciones de Guaranies en el Paraguay. Asunción, CEPAG.

Melià, Bartomeu. 1992. La lengua guaraní del Paraguay. Madrid, Mapfre.

Melià, Bartomeu. 2000. "Un guaraní reportero de guerra”. En: Acción. Revista Paraguaya de Reflexión y Diálogo, N²08, [En línea]: http://www.uninet.com.py/accion 
Melià, Bartomeu. 2006. "Escritos guaraníes como fuentes documentales de la historia paraguaya”. En: Nuevo Mundo/Mundos Nuevos [En línea] Debates, puesto en línea el 5 abril 2006, http://nuevomundo.revues.org/2193

Melià, Bartomeu. 2011. "La lengua transformada: el guaraní en las Misiones Jesuíticas". En: Wilde, Guillermo (ed.). Saberes de la conversión. Jesuitas, indígenas e imperios coloniales en las fronteras de la cristiandad. Buenos Aires: Editorial SB, pp. 81-98.

Neumann, Eduardo Santos. 2005. Práticas letradas guaraní: produçâo e usos da escrita indígena (séculos XVII e XVIII). Tesis doctoral. Universidade Federal do Río de Janeiro, [En línea]: http://www.dominiopublico.gov.br/pesquisa/DetalheObraForm.do?select action $=\&$ co obra $=107879$

Neumann, Eduardo Santos. 2007. "A escrita dos guaranis nas reduçôes: usos e funçôes das formas textuais indígenas. Século XVIII". En: Topoi - Revista de História, N 15, Río de Janeiro, Universidad Federal de Río de Janeiro, pp. 49-79 [En Línea]: http://www. revistatopoi.org/numeros_anteriores/topoi15/topoi\%2015\%20-\%20artigo3.pdf

Ruiz de Montoya, Antonio. 1639 [1989]. Conquista espiritual hecha por los religiosos de la Compañía de Jesús, en las provincias del Paraguay, Paraná, Uruguay y Tape. Estudio preliminar y notas de Ernesto J. A. Maeder, Rosario, Equipo Difusor de Historia Iberoamericana.

Ruiz de Montoya, Antonio, 1639 [2011]. Tesoro de la lengua guaraní. Asunció, CEPAG.

Ruiz de Montoya, Antonio. 1640 [1994]. Arte y bocabulario de la lengua guaraní. 2 T., Madrid, Ediciones de Cultura Hispánica, Agencia Española de Cooperación Internacional, Edición crítica a cargo de Silvio Liuzzi con edición faccimilar de 1640.

Obermeier, Franz. 2006. „Der argentinische Erstdruck Nierembergs ,De la diferencia“ in Guarani im Kontext der Bilderzyklen in Lateinamerika im 18. Jahrhundert" [En línea] http://archiv.ub.uni-heidelberg.de/artdok/volltexte/2006/154/

Obermeier, Franz. 2011. „Wie und mit welchen Mitteln erforschte man südamerikanische Indianersprachen“. En: Wolfenbütteler Notizen zur Buchgeschichte, $\mathrm{N}^{\circ} 35$, Vol 2, Harrassowitz Verlag, , pp. 167-183.

Palomera Serreinat, Lluís. 2002. Un ritual bilingüe en las reducciones del Paraguay: el manual de Loreto (1721), Cochabamba, Verbo Divino.

Pastells, Pablo y Francisco Mateos [Org.]. 1918-1949. Historia de la Compañía de Jesús en la provincia del Paraguay, 8 T., Consejo Superior de Investigaciones Cientificas, Instituto Santo Toribio de Mogrovejo, Madrid.

Peramás, José Manuel. 1768 [1952]. José Manuel Peramás y su diario del destierro. Editado por Guillermo Furlong, Buenos Aires: Librería del Plata.

Restivo, Paulo. 1722 [1893] Lexicon Hispano-Guaranicum "Vocabulario de la lengua Guarani" inscriptum a Paulo Restivo secundum Vocabularium Antonii Ruiz de Montoya anno 1722 [...] [Edición de Christianus Fredericus Seybold], Stuttgard, Kohlhammer. [En línea]: archive.org.

Restivo, Paulo. 1724 [1892] Linguae Guarani Grammatica Hispanice a Rev. P. Jesuita Paulo Restivo secundum libros Antonii Ruiz de Montoya, Simonis Bandini aliorumque adjecto Particularum lexico anno MDCCXXVIII in Civitate S. Mariae Majoris [...] [Edición de Christianus Fredericus Seybold]. Stuttgart, Kohlhammer. [En línea] archive.org.

Sebastián, Santiago 1992, "Lectura iconográfica de la versión guaraní del libro del padre Nieremberg 'De la diferencia entre lo temporal y eterno", en: Boletin del Museo e Instituto Camón Aznar, Nr. 48/49, Zaragoza, Instituto Camón Aznar, pp. 309-328.

Thun, Harald. 2003. "La evolución de la escripturalidad entre los indígenas guaraníes" En: Rodrijuelo, Emilio y María Fuentes (ed). Simposio Antonio Tovar sobre lenguas amerindias. Valladolid, Universidad de Valladolid, pp. 9-23

Thun, Harald. 2008a. "La hispanización del guaraní jesuítico en 'lo espiritual'y en 'lo temporal'. Primera parte: El debate metalingüístico". En: Thomas Stehl (Org), Kenntnis und Wandel 
Cerno - Obermeier. Lingüística e investigación de documentos en guaraní (siglo XVIII).

der Sprachen. Beiträge zur Potsdamer Ehrenpromotion für Helmut Lüdtke. Tübingen, Gunter Narr Verlag, pp. 217-240.

Thun, Harald. 2008b. "La hispanización del guaraní jesuítico en 'lo espiritual'y en 'lo temporal'. Segunda parte: Los procedimientos". En: Dietrich, Wolf y Haralambos Symeonidis (ed), Geschichte und Aktualität der deutschprachigen Guarani-Philologie. Berlín, Lit Verlag, pp. 141-169

Thun, Harald. En prensa. "El manuscrito Güllich". Contribución a las XIV Jornadas Internacionales sobre las Misiones Jesuiticas, Universidad Técnica Particular de Loja, Agosto 7-12 2012; San Ignacio de Velasco, Bolivia.

Wilde, Guillermo. 2012. Religión y poder en las Misiones de Guaranies. Buenos Aires: Sb. 